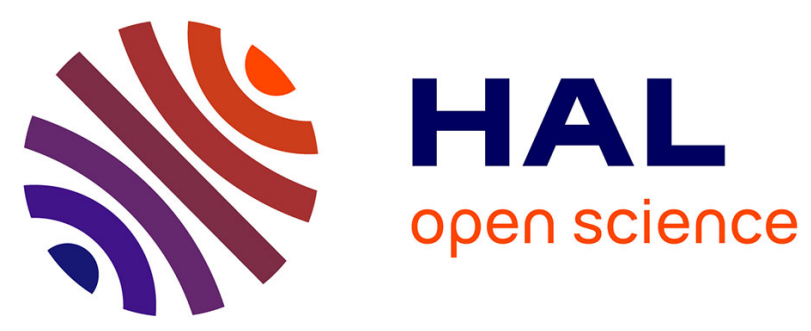

\title{
The effects of graphical overviews on exploratory behaviour and knowledge acquisition in hypertext environments
}

Ton de Jong, Anja van Der Hulst

\section{- To cite this version:}

Ton de Jong, Anja van Der Hulst. The effects of graphical overviews on exploratory behaviour and knowledge acquisition in hypertext environments. Journal of Computer Assisted Learning, 2002, 18 (2), pp.219- 231. 10.1046/j.0266-4909.2002.00229.x . hal-00190678

\section{HAL Id: hal-00190678 \\ https://telearn.archives-ouvertes.fr/hal-00190678}

Submitted on 23 Nov 2007

HAL is a multi-disciplinary open access archive for the deposit and dissemination of scientific research documents, whether they are published or not. The documents may come from teaching and research institutions in France or abroad, or from public or private research centers.
L'archive ouverte pluridisciplinaire HAL, est destinée au dépôt et à la diffusion de documents scientifiques de niveau recherche, publiés ou non, émanant des établissements d'enseignement et de recherche français ou étrangers, des laboratoires publics ou privés. 
Author Posting ( $\odot$ Blackwell Publishing Ltd. This is the author's version of the work. It is posted here by permission of Blackwell Publishing Ltd. for personal use, not for redistribution. The definitive version was published in the Journal of Computer Assisted Learning, Volume 18, Issue 2, Page 219-231. http://dx.doi.org/10.1046/j.02664909.2002.00229.x

The effects of graphical overviews on

knowledge acquisition in hypertext

Ton de Jong

Faculty of Educational Science and Technology

University of Twente

The Netherlands

Anja van der Hulst

TNO FEL

The Hague

The Netherlands 


\begin{abstract}
One of the main and recognized problems of learning with hypertexts is that learners are easily distracted and end up 'lost in hyperspace'. As a result, they do not acquire complete and adequate knowledge. In this study, we enhanced a hypertext environment with a graphical overview that represented the basic structure of the domain and we designed the layout in such a way that learners were unobtrusively encouraged to follow a sequence of exploration that followed the domain structure. This so-called 'visual' lay-out was compared with two lay-outs that presented randomly positioned nodes. One of these two lay-outs contained hints (using 'highlighting') to stimulate learners to follow a domain related exploration similar to the one incorporated in the visual lay-out. The other (control) lay-out did not provide such hints. Results showed that participants from both the 'visual' and the 'hints' conditions showed a more domainrelated exploration pattern than participants from the control condition. Participants in the 'visual' lay-out did not show a better recall of the content of the nodes as such, but showed a significantly better acquisition of knowledge of structure than participants from the other two conditions. These data indicate that a visual display conveys knowledge in its own right and that knowledge gained does not depend on the exploration route followed in the hypertext material.
\end{abstract}

1. Introduction

Exploratory learning environments, such as hypertext or hypermedia based learning environments, are expected to engage 
learners in an active learning process. Many authors claim that this should lead to better motivation, comprehension, and transfer (Dillon \& Gabbard, 1998; Jacobson \& Archodidou, 2000). Review studies, however, assessing the effect on learning from hypermedia have shown no overall effects compared to linear presentation formats (see e.g., chen \& Rada, 1996; Dillon \& Gabbard, 1998; Tergan, 1997) and some studies even show detrimental effects of the use of hypertext or hypermedia (see e.g., McKnight, Dillon, \& Richardson, 1990; Rada \& Murphy, 1992). A major barrier for fruitful exploration learning in hypertexts as signaled by several authors (e.g., Conklin, 1987, Foss, 1987; Marchionni, 1988; Rouet, 1990) is that people easily 'get lost in hyperspace'while exploring. Hypertext offers a large degree of freedom and users seem to have trouble handling this freedom. Early in the research on hypertext, Foss (1987) referred to the 'art museum phenomenon'. that is, people having spent long days, wandering around, gazing at paintings, may recall details but still have failed to discover the main lines. Disorientation, or 'losing context', according to Conklin (1987), is a problem that is endemic to the use of hypertext. With traditional, linear, instructional text the situation is different. This type of text is generally specifically organized for learning. The learner does not have to decide about the sequence in which to learn the nodes, and usually overviews are provided in the form of abstracts and summaries. In contrast, exploration of a hypertext document does require the learner to perform complex cognitive actions such as planning and monitoring. It is 
likely that the observed ineffectiveness of learning in exploratory environments arises from an inability to meet the cognitive demands made by the exploratory environment. Learner activity is assumed desirable, but in plain exploratory environments, particularly in the more extensive ones, too much seems to be left to the learner.

Various types of solutions have been proposed to help learners to overcome the complexity of exploration. Quite restrictive support measures are the 'guided tours' as used by Allison and Hammond (1989) and Arents and Bogaerts (1993). More freedom is given by support that helps to monitor the history of the exploration, e.g., by leaving 'footprints' (Foss, 1987; McAleese, 1989) sometimes combined with means to mark important sections (Rouet, 1990) and annotating the material studied (Monk, 1990). Graphical overviews have taken lead in helping users overcoming disorientation (see e.g., Conklin, 1987; McAleese, 1989; de Young, 1990). Several studies have found that graphical overviews, and even the specific layout of the graphical interface, affects the effectivity and efficiency of information retrieval from the hypertext both under general conditions (Mogaheg, 1992; McDonald \& Stevenson, 1998) and in performing a specific (design) task (de Vries \& de Jong, 1997; 1999). In a metaanalysis of 23 studies, Chen and Rada (1996) concluded that the most significant result concerned the affect of graphical overviews on results gained. Norman (1988) argues that one of the main principles for interface design is 'visibility' (i.e., the internal functional structure of a system must be 
visible at the outside to allow the user to make deliberate decisions on how to handle the system). In line with Norman, we assume that the need for visibility also holds for an internal structure of a domain that is to be learned from a hypertext

The effects of a visual display of the domain structure on learning from hypertext may come in two ways. First, providing learners with a systematic visual overview of the domain is expected to induce a systematic route through the domain and may thus lead to a better acquisition of the structure of the domain. This latter claim is supported by studies on the effects of the sequence in which written material is presented on learning. Many of those (older) studies compared randomly generated sequences with sequences that are based on the logical structure of the domain. Although several early studies did not report any differences (Vlachouli-Roe, Case, \& Roe, 1962; Levin \& Baker, 1963), a number of later studies reported a significantly better acquisition of knowledge when following domain structure related sequences (Brown, 1970; Tobias, 1973; Kintsch, Mandel, \& Kozminsky, 1977). Dee-Lucas and Larkin (1995) state that, compared to traditional text, hypertext systems make it more difficult for learners to build an integrated representation of the information because they move between units, missing the continuous line of reading that is characteristic of traditional text. Second, visual overviews are supposed to enhance acquisition of domain structure because they display the structure of the domain directly. Whereas in traditional text learners have to extract 
the overall structure (relations between higher and lower level ideas) from the running text, visual overviews display these structures directly in the titles of nodes and their interrelations. Dee-Lucas and Larkin (1995) compared learning from a hypertext with a visual overview with learning from the same hypertext with names of nodes presented as an unstructured list. They found that learners with the unstructured overview produced less coherent representations than learners with the graphical, structured overview. In another study, however, Jonassen (1993) found that students who were provided with a graphical overview did not acquire better 'structural knowledge' than students who learned with unstructured nodes or with an interface that lead them on a node-by-node basis through the hypertext. Jonassen (1993) even found on a recall test that the group learning with the unstructured interface outperformed the groups working with the structured interfaces.

In the present study, we investigated the effects of presenting a visual display of the domain structure in a hypertext system on knowledge acquisition. The visual display we used in our study was comparable to the visual or graphical displays from the Jonassen (1993) and Dee-Lucas and Larkin (1995) studies. We have extended these two studies in two ways. First, we have measured the learning results in three different ways: knowledge of the overall structure of the domain (configural knowledge), knowledge of the relations between two specific nodes (propositional knowledge), and knowledge of the content of the nodes (definitional 
knowledge). Jonassen concentrated on what we have called here propositional knowledge and definitional knowledge, whereas the assessment used in the Dee-Lucas and Larkin (1995) study (an analysis of summaries made by students) seems to emphasize what we have called configural knowledge. Second, we have made an analysis of the exploration behavior of learners that went beyond measuring nodes visited and time spent at each node and that tried to capture their exploration pattern.

These extensions made it possible to test some specific hypotheses. We first tested the idea that learners exploring a hypertext system in which the structure of the domain is displayed visually, will show a better acquisition of knowledge of overall structure (configural knowledge) compared to learners who explore a hypertext that lacks this visual display of the domain. We further tested the idea that acquisition of this overall structure is caused by the direct visual information and that knowledge of relations between nodes (propositional knowledge) results from a structured exploration pattern. The final assumption is that learning the content of a node (definitional knowledge) is independent of the form of the visual display and the sequence of exploration.

\section{Method}

\subsection{The Domain}

In the selection of the subject matter for this experiment, the prior knowledge of the learners was a crucial factor. We assume that the effectiveness of the interface 
manipulation depends on the amount and nature of the learner's prior knowledge with the structure of the interfaces most likely having more effect with subject matter material that is relatively unfamiliar to the learners. Studies by Mohageg (1992), McDonald and Stevenson (1998), and Dee-Lucas (1999) showed that experts rely on their prior knowledge when interacting with hypertext system, whereas novices more rely on cues in the interface. Following this, we chose a domain that was largely unfamiliar to participants in our study. The hypertext system consisted of 26 hypertext fragments in the domain of fuel supply systems of a Dutch automobile. The fragments contained descriptions of the various aspects of process control in a modern fuel injection system. In such systems, several factors are measured (e.g., external temperature) to supply an optimal amount of fuel for a combustion engine to run properly. For example, in cold weather conditions, the system needs to supply relatively more fuel as more will condense in the engine and is therefore not available for combustion. The hypertext was organized in a hierarchical way. At the highest level, it contained general introductions to measure and control processes. At the subsequent level, it provided descriptions of the particular processes in a fuel supply system. Finally, at the lowest level, it gave examples of measuring or control under specific circumstances. 


\subsection{Experimental Design}

A hypertext system was developed with three different interfaces.

In Condition 1, the 'visual' condition, the overview was organized using a 'visual' layout. That is, we enhanced a hypertext environment (on 'fuel supply systems') with a graphical overview that represented the basic structure of the domain and we designed the layout in such a way that learners were unobtrusively encouraged to choose a sequence of exploration that followed the domain structure. This lay-out followed a vertical hierarchical structure of nodes with is-a relations (e.g., a motor temperature measurement is-a measurement), the top two horizontal levels indicated sequential, temporal relations (e.g., a motor temperature measurement is_followed_by a determination of default signal), and the lower three horizontal levels indicated causal relations (e.g., measurement of a high motor temperature causes relatively low signal correction). An impression of the interface of the 'visual' condition is given in Figure 1, but only a limited number of the nodes and relations are presented.

In Condition 2, the 'hints' condition, also a visual layout was used to present the same nodes as in the visual display of Condition 1 , but now the nodes were arranged randomly on the screen (without nodes overlapping). In Condition 2, participants were provided with hints on how to traverse the system. These hints were implemented by highlighting nodes in the overview, where the sequence of 
highlighting reflected the domain-related layout from Condition 1 .

Condition 3, the 'control' condition, was created by using the same 'random' overview as was used in condition 2. In this case, no hints on the sequence of traversing the system were provided.

Insert Figure 1 about here

Since our aim was to study the influence of the displayed structure of the domain on learning we decided to provide as little textual information concerning relations as possible. Therefore, great care was taken to avoid explicit references within text fragments to other nodes. Hence, the only information on relations was provided by the visualization of those relations in the graphical overview. This visualization was the only access to the hypertext fragments and thus, though unusual, it was not possible to go from within one hypertext fragment directly to the other. Finally, in the interface the learners were allowed to move the nodes, primarily because moving usually made relations between nodes more evident. It should be noted that in all conditions participants were fully autonomous to decide on the sequence of exploration.

\section{3 Participants}

The participants ( $\mathrm{N}=46 ; 15$ in the 'visual' condition, 15 in the 'hints' condition, and 16 in the 'control' condition) 
were first year undergraduates in Psychology. Participation in the experiment was part of a study requirement, though participants had the right to choose from several experiments. The participants were assigned randomly to the conditions, with the exception that both genders were equally distributed over conditions.

\section{4 Procedure}

Prior to working with the hypertext system, the participants were trained on a practice domain consisting of four nodes. Dee-Lucas and Larkin (1995) showed that the influence of the form of the overview of a hypertext on participants' performance is higher when learners receive global learning goals compared to when they receive specific learning goals. Participants received a global assignment asking them to learn as much as possible from the hypertext. They were also informed that an above moderate performance on the post-test would be (financially) rewarded. The participants were instructed to work at least 25 minutes with the hypertext and no maximum time limit was set. Participants were instructed that all text fragments should be read and each fragment could be read more than once. Differences in efficiency between conditions could emerge since the total time was not limited and we allowed participants to visit text fragments more than once. To get a clear picture of the exploratory trajectory, participants were forced to read only one fragment at a time. Finally, three types of knowledge 
tests (as described later) were administered at two points in time: prior to and after the session with the hypertext.

\subsection{Data Collection}

\subsubsection{Process Measures}

All actions of the participants were logged with a time stamp. This provided us with information on the overall activity in the environment, time spent, and nodes visited. The logfiles were also used to extract the exploration pattern of the participants. First, we looked at the 'connectivity' of the participants' exploration. More specifically, we looked at the number of 'jumps' made by participants. That is, did they follow links in the interface or did they traverse between unrelated nodes? Second, we looked at the 'consistency' of traversing. That is, did the exploration pattern follow the hierarchy, temporal, and causal parts of the domain structure? We choose to use logfiles since these data can be gathered unobtrusively and this does not interfere with the learning process.

\subsubsection{Product Measures}

We have claimed that differences in the sequence of exploration would mainly affect the acquisition of knowledge of structure and not the learning of the content of the nodes. Content of the nodes was measured by a definitional knowledge test. Knowledge of structure was first measured with a test of knowledge of direct relations between concepts (the propositional knowledge test). Second, knowledge of the 
overall structure of the domain was measured by the so-called 'configural' knowledge test (cf., Goldsmith, Johnson, \& Acton, 1991) .

Definitional knowledge. Definitional knowledge was operationalized as knowledge of concepts acquired from the text fragments. This type of knowledge was tested by means of a multiple choice test that required reproduction of facts as given in the text fragments that described those concepts. An example item from the definitional knowledge test is: "The position of the throttle switch provides information on: 1) the amount of oxygen supplied; 2) the amount of fuel supplied; $3)$ both 1 and 2 are correct; 4) both 1 and 2 are incorrect; 5) no idea". The final answer option was in all cases 'no idea', this option was added since we anticipated little knowledge of the subject matter, especially in the pre-test. By instructing the participants not to guess but to use this option we hoped to avoid too large a bias due to guessing. Pre-test and posttest test both consisted of a total of 20 items with 5 possible answers.

Propositional knowledge. For measuring propositional knowledge, we developed several types of items. To test for hierarchical (i.e., generalization-specification) relations, we asked for categorization, or for identification of subclasses. Temporal relations were tested by asking for ordering in time or by asking for the missing parts of a procedure. Causal relations were tested by asking for 
predictions. All relations that were present in the hypertext system (except for the absolutely trivial ones) were tested. An example item asking for a temporal relation is: "The process of correction for external influences is amongst other things preceded by measuring: 1) the motor temperature; 2) the RPM (rotations per minute); 3) the air pressure in the inlet; 4) none of the above options is correct; 5) no idea". An example item asking for a causal relation is: "A malfunction in the motor temperature sensor during cold start causes the blocks of the basic supply signal to be: 1) too short; 1) too long; 3) irregular; 4) none of the above options is correct; 5) no idea". Pre-test and post-test both consisted of a total of 20 items, each with 5 answer alternatives, where again one of the options was the 'no idea' option.

Configural knowledge. Configural knowledge was tested by means of a card sorting task (see Shavelson \& Stanton, 1975). In this card sorting task, the participants were instructed to cluster the 26 nodes of the hypertext. For this task, a tool was created with an interface identical to that of the hypertext system, except that it did not allow access to the hypertext fragments. Participants were instructed to move the nodes to form stacks that were clusters of related nodes. Neither clustering criteria nor limit on cluster-size were given. To obtain a measure for knowledge of the overall structure, the results of each participants' card sorting task were compared with a norm model. This norm model was chosen so that it resembled the original domain organization as much as 
possible. To calculate the correspondence to the norm clustering, a proximity matrix was generated from both the participant and the norm clustering. A cell of the proximity matrix is filled with a 1 if row and column concepts belonged to the same cluster, and with $\odot$ if they belonged to different clusters. The correspondence of the participants' proximity matrix to the norm matrix was calculated using a measure for correspondence by de Jong and Ferguson-Hessler (1986). This measure accepts matrices composed of dichotomous data as input .

\subsubsection{Predictions}

In Condition 1, the 'visual' condition, following domainrelated traverses is induced by the domain-related visual display whereas in Condition 2, the 'hints' condition, a domain related route is stimulated by the hints that are presented. The first prediction therefore was: Participants in Condition 1 (the 'visual' condition) and Condition 2 (the 'hints' condition) will follow a more domain-related sequence of traversing the hypertext system than participants in Condition 3 (the 'control' condition).

Following the first prediction, participants Conditions 1 and 2 would follow a domain-related sequence of exploration that would be absent in Condition 3. Following a domainrelated route means that traverses follow the named links between nodes. As a consequence, we predicted that participants in Condition 1 (the 'visual' condition) and Condition 2 (the 'hints' condition) would acquire higher gains 
on the propositional test than the participants in Condition 3 (the 'control' condition).

The domain related sequence of exploration in Conditions 1 and 2 also should lead to a better knowledge of the overall structure of the domain. In Condition 1 , this overall structure is not only expressed in the links between nodes but is also directly displayed by the lay-out. We, therefore, predicted that participants in Condition 1 (the 'visual' condition) would gain better configural knowledge than participants in Condition 2 (the 'hints' condition), who again would acquire better configural knowledge than the participants in Condition 3 (the 'control' condition).

Since learning the content of node is independent of the overall display and of the sequence of exploration we predicted that participants in all conditions would gain the same amount of definitional knowledge.

\section{Results}

\subsection{Process Measures}

\subsubsection{Time on Task and Overall Activity}

Analysis of variance revealed no differences between conditions for time on task $(\underline{F(2,43)}=2.49$, ns $)$. The mean duration in the hypertext system in seconds for the 'visual' group was 1691 ( $\underline{\mathrm{SD}=253})$, the 'hints' group spent 1990 seconds in the hypertext $(\underline{S D=439})$, and the control group 1833 seconds $(\mathrm{SD}=482)$. Activity in terms of the number of traverses also showed no differences $(F(2,43)=.85, n s)$. The mean number of traverses in the 'visual' group was 41.60 ( $\underline{\mathrm{SD}=12.62}), 35.13$ 
$(S D=16.03)$ in the 'hints' group, and $43.56(\underline{S D=24.76})$ in the control group. No correlation was found between time on task and the dependent variables propositional knowledge $(r=-.03)$ or configural knowledge $(\underline{r=.02})$.

\section{1 .2 Connectivity}

The first measure of the participants' traverse pattern was called connectivity. To assess connectivity, we calculated for each participant the average distance covered in visiting two nodes that were related in the hypertext system. The distance $\underline{d}$ of a traverse was determined by means of calculating the distance covered to get from node $\underline{A}$ to a the next node in the sequence, entity $B$. The distance between entities $A$ and $B$ was defined as the number of relations of the domain structure to be traversed to get from $A$ to $B$ via the shortest path. This was corrected for 'necessary' traverses, e.g., if one ended up in the leaves of a fully explored branch. Connectivity was expressed as $1 / \mathrm{d}$.

The connectivity scores showed a main effect for condition $(\underline{F(2,43)=11,32}, \underline{p<.00})$, where a post-hoc analysis (Scheffé) revealed that connectivity scores were significantly higher for both 'visual' $(1 / d=.90 ; s d=.04)$ and 'hints' group $(1 / d$ $=.87 ; \mathrm{sd}=.10$ ) when compared to those of the control group $(1 / d=.75 ; s d=.13)$. This means that in their exploration patterns participants in the 'visual' and 'hints' groups more closely followed the links from the interface than participants in the control group.

During a qualitative analysis of the traverses of 12 
participants from the 'visual' and control group (the three best and three worst performers from both groups), we observed differences between the groups in the initial exploration phase. The trajectory of the 'visual' group was found to be highly connective from the beginning. Large 'jumps' were only observed in a later stage of exploration. In contrast, within the control group 'jumping' was certainly seen in the initial stage. A possible explanation might be that participants in the control group started browsing the material but that the 'visual' group skipped this phase. To investigate this, each trajectory was split up into an 'initial phase', incorporating the first 10 traverses and a 'final phase' incorporating the traverses 11 and on. The connectivity results for both phases can be found in Table 1 .

Insert Table 1 about here

The connectivity scores for the control group were significantly lower in the initial phase $(t=-5.17, \underline{d f=15}$, $\mathrm{p}<.00$ ) compared to the final phase. In addition, the connectivity scores of the 'visual' group were significantly higher in the initial phase $(\underline{t=2.37}, \underline{\mathrm{df}=14}, \underline{\mathrm{p}<.02})$ compared to the final phase, while the same holds (marginally) for the 'hints' group ( $\mathrm{t=1.64}, \mathrm{df}=14, \underline{\mathrm{p}} \mathbf{. 0 6})$.

\subsubsection{Consistency}

In addition to whether participants followed links in the hypertext, we measured if they followed the domain structures 
in the interface. As indicated above, the hypertext system basically consisted of a number of structures, one (vertical) hierarchical structure, two (horizontal) temporal structures, and three (horizontal) causal structures. For each of these structures, we determined a measure of consistency. For each exploration of a separate structure, we determined whether it followed the links in the structure or not. This was done using a pre-defined number of links for each structure, and assessing the exploration with a $\odot$ if the number of links was lower than the pre-defined number and a 1 if this was equal or higher than the pre-defined number. Table 2 presents the average consistency scores for the separate structures in the different experimental groups.

Insert Table 2 about here

The data on consistency (see Table 2) showed an overall effect for condition, except for the first causal structure. Analysis of variance revealed the following effects: for hierarchy $(\underline{F(44,2)=45.48}, \underline{p<.00})$, temporal-1 $(\underline{F(44,2)=4.74}$, $\underline{\mathrm{p}<.01})$, temporal-2 $(\mathrm{F}(44,2)=13.81, \underline{p}<.00)$, causal-1 $(\underline{F(44,2)=1.70}, n s)$, causal-2 $(\underline{F(44,2)=16.52}, \underline{p<} .00)$, causal -3 $(\mathrm{F}(44,2)=12.25, \underline{p}<.00)$. Post hoc analysis revealed that level of consistency in the 'visual' and 'hints' conditions is for all primitive structures significantly higher than in the control condition, again with the exception of the first causal structure. 


\section{2 Product Measures}

The results of the test for definitional and propositional knowledge are expressed in terms of the number of items correct (out of 20), whereas configural knowledge is expressed in terms of correspondence to a norm model. For the data on configural knowledge, due to lack of activity in the card sorting task, the results of one participant from the 'visual' condition and that of one participant from the hints condition had to be removed from the data set $(N=44)$. Table 3 and Figure 2 present an overview of the results.

Insert Table 3 about here

As can be seen in Table 3, no large differences in prior knowledge were found. One way multivariate analysis of variance did not reveal a difference between conditions $(F(6,80)=1.20, n s$.$) for the pre-test results. Although the$ pre-test differences for propositional knowledge were small, disregarding these may lead to inappropriate conclusions. Hence, from here on we will use 'knowledge gain' scores that reflect the differences between pre-test and post-test scores. These knowledge gain scores, when subjected to a multivariate analysis, showed an overall constant effect, indicating that the participants indeed learned from working with the hypertext environment $(\underline{F(3,41)=64.87}, \underline{p<.00})$. This learning effect was significant for definitional $(F(1,43)=167.25$, $\underline{\mathrm{p}<.00})$, propositional $(\underline{\mathrm{F}(1,43)=122.30}, \underline{\mathrm{p}<.00})$ and configural knowledge gain $(\underline{F(1,43)=31.56, \underline{p}<.00})$. The different 
treatments were found to affect learning since the gain scores showed a main effect of condition $(\underline{F(6,78)=3.76}, \underline{p<.00})$. Using a univariate analysis, this main effect can be ascribed to effects on propositional and configural knowledge. For these types of knowledge gain, the results revealed significant differences between the conditions $(\underline{F(2,41)=7.46}, \underline{p<.00})$ and $(\underline{F(2,41)=6.81}, \underline{p<.00})$, respectively. Univariate analysis revealed no differences for definitional knowledge $(F(2,41)=.59)$. The effects on propositional and configural knowledge were analyzed using a one-way univariate analysis for each of the dependent variables. Post hoc analysis (Scheffé) revealed that differences between the 'visual' condition and the 'hints' condition and differences between 'visual' condition and control condition were significant (at $\alpha=.05)$ for both the propositional knowledge and the configural knowledge test. Differences between the 'hints' and the control conditions were not significant for propositional knowledge. Differences between the 'hints' and the control conditions were only marginally significant $(\underline{t=1.67,} d f=28$, $\underline{\mathrm{p}<.06}$ ) for configural knowledge. For definitional knowledge, there were no significant differences between conditions.

Insert Figure 2 about here

4. Conclusions and discussion

In the present study, we assessed the impact of different interfaces on learning from a hypertext system. The study was designed in such a way that effects from the graphical 
interface and the learning route induced by the interface could be separately assessed. Following critical comments from review studies (Dillon \& Gabbard, 1998; Tergan, 1997), we tried to use a set of knowledge measures directly tapping types knowledge relevant for the stated theoretical hypotheses. Using the logfiles, we were also able to assess the learning process in a deeper manner than has been done in previous research.

We first tested the idea that the interface may influence the exploration route that students follow in a hyptertext system. The related prediction in our study was that in presenting a visual overview of the domain (Condition 1 , the 'visual' condition) and giving learners hints to follow a domain related route (Condition 2, the 'hints' condition) would encourage participants to follow a more domain related sequence of traversing the domain than participants in a condition that lacked these features (Condition 3, the control condition). Data showed that participants in the 'visual' and 'hints' conditions indeed followed the links in the interface (displaying the domain structure) more closely than participants in the control condition. A further comparison of the 'visual' with the control condition showed that in the control condition jumping through the nodes was especially dominant at the start of the learning process. Process data further showed that, overall, participants from the 'visual' and 'hints' conditions were more consistent in following the separate 'sub-structures' in the hypertext than participants from the control condition. In summary, we found that 
participants in the 'visual' condition, in which the structure of the domain was visually displayed, and participants in 'hints' condition, in which the structure of the domain was suggested by highlighting relations, showed a more domainrelated exploration pattern than participants in the control condition. This finding supports the general idea that interface aspects can influence learners' exploration routes.

Our second prediction stated that participants in the two conditions that guided the exploration route ('visual' and 'hints' conditions) would show higher gains on the propositional test than participants in the control condition. The basic idea was that propositional knowledge test assessed the relations between separate concepts and that in the 'visual' and 'hints' conditions this would be positively affected by the domain related route that was followed. Data partly followed this prediction. Only participants in the 'visual' condition had higher gains on propositional knowledge than participants in the control condition. There was no significant difference in gain between participants from 'hints' and control condition. As was tested under our first prediction, we did not find differences in the exploration route between participants in the 'visual' and 'hints' conditions. Thus, the conclusion here can be that it is not the exploration route per se that leads to the acquisition of propositional knowledge.

The third prediction stated that participants in the 'visual' condition would gain higher configural knowledge than participants in 'hints' condition, who in turn would show a 
higher gain in configural knowledge than participants in the control condition. We expected this effect based on the idea that the visual overview directly displayed the configuration of the domain. Following a domain related exploration route (as was expected in both the 'visual' and 'hints' condition) would provide learners with the opportunity to experience the overall configuration of the domain but less directly than in the visual overview. This prediction was partly corroborated by the data. The difference between the 'visual' and 'control' conditions was significant. The difference between the 'hints' and the 'control' conditions almost reached significance.

The final prediction that conditions would show no differences in knowledge of the content of nodes was supported by the data. This confirmed the idea that what students learned within a node was independent from the route that brought them there or the context in which it was embedded.

The outcomes of the present study help us to better understand results from previous studies and it underscores the value of recommendations done by several review studies. From the results we may also learn about the theoretical underpinnings of learning from hypertext and find inspiration for the design of hypertext environments.

One of the main outcomes of our study is that different results can be found for different types of knowledge measures. This explains the seemingly contradictory results of the Jonassen (1993) and Dee-Lucas and Larkin (1995) studies. Using dependent measures that resembled our definitional and 
propositional test, Jonassen found no advantage to providing students with graphical overviews or node-by-node guidance. When Dee-Lucas and Larkin used a dependent measure similar to our configural test, they found positive effects for a graphical overview compared to a textual unstructured overview. This is in line with the results of the present study. In a more general sense, this touches upon the conclusion drawn in several review studies on learning from hypermedia (Chen \& Rada, 1996; Dillon \& Gabbard, 1998; Tergan, 1997) that the results of studies reviewed are equivocal. The results of the present study support the notion that learning from hypertext may occur, though we did not compare it to more linear types of learning material. Dillon and Gabbard (1998) concluded from their review of studies comparing learning from hypermedia and other media that hypermedia is most useful when learning complex and extensive domains where searching for information is relevant and when data manipulation and comparison of data is important. In our study, the domain was not an extensive domain, but due to the different types of relationships involved and the nature of the domain it could be seen as not an easy topic for our participants. Of course, we can only speculate about effects when a more extensive domain is involved but we would expect, following Dillon and Gabbard, that the effect of a graphical interface would then even be more distinctive.

One of the most cited theoretical notions used in the context of learning from hypertext is cognitive flexibility theory (e.g., Spiro \& Jehng, 1990; Spiro, Feltovich, Jacobson, 
\&, Coulson, 1992). Basic to this notion is the idea that approaching a domain from different angles enables learners to make multiple comparisons and relations and this leads to a 'flexible' cognitive structure. Though we did not test this idea directly, the implicit assumption in cognitive flexibility theory that knowledge gained is influenced by the route followed is not supported in our study. The overall conclusion from this study is that the most significance influence on learning results comes from the graphical overview and that the effect of this interface is due to the direct display of the domain structure. Designing hypermedia environments should therefore not only look at the overall exploration pattern of learners but present the learner with graphical overviews and link these to deep representations of the domain. Recent and detailed approaches towards designing hypermedia environments (Jacobson \& Archodidou, 2000) take this into account.

\section{References}

Allison, L., \& Hammond, N. (1989). A learning support environment: The hitchhiker's guide. In. R. McAleese (Ed.), Hypertext: Theory into practice ( $p$ p. 62-74). 0xford: Intellect Limited.

Arents, H., \& Bogaerts, W. (1993). Navigation without links and nodes without contents: Intentional navigation in a third order hypermedia system. Hypermedia, $\underline{5}(3)$, 187-204. 
Chen, C., \& Rada, R. (1996). Interacting with hypertext: A meta-analysis of experimental studies. Human-Computer Interaction, 11, 125-156.

Brown, J. (1970). Effects of logical and scrambled sequences in mathematical materials in learning with programmed instructional materials. Journal of Educational Psychology, 61, $41-45$.

Conklin, J. (1987). Hypertext: An introduction and survey. IEEE Computer, 20, 17-41.

Dee-Lucas, D. (1999). Information location in instructional hypertext: effects of content domain expertise. Proceedings of Ed-Media 99. Seattle, WA: AACE.

Dee-Lucas, D., \& Larkin, J.H. (1995). Learning from electronic texts: effects of interactive overviews for information access. Cognition and Instruction, 13, 431-468.

Dillon, A., \& Gabbard, R. (1998). Hypermedia as an educational technology: A review of the quantitative research literature on learner comprehension, control and style. Review of Educational Research, 68, 322-349.

Foss, C. (1987). Effective browsing in hypertext systems. (CeRCLe Technical report $\mathrm{nr} .41$ ). Lancaster: Department of computing, University of Lancaster.

Goldsmith, T., Johnson, P., \& Acton, W. (1991). Assessing structural knowledge. Journal of Educational Psychology, 83, $88-96$.

Jacobson, M. J., \& Archodidou, A. (2000). The design of hypermedia tools for learning: Fostering conceptual change and transfer of complex scientific knowledge. Journal of 
the Learning Sciences, 9, 145-199.

Jonassen, D.H. (1993). Effects of semantically structured

hypertext knowledge bases on users' knowledge structures.

In C. McKnight, A. Dillon \& J. Richardson (Eds.),

Hypertext, A psychological perspective (pp. 153-169). New York: Ellis Horwood.

de Jong, T., \& Ferguson-Hessler, M. (1986). Cognitive structures of good and poor novice problem solvers in physics. Journal of Educational Psychology, 78, 279-288.

Kintsch, W., Mandel, T.S., \& Kozminsky, E. (1977). Summarizing scrambled stories. Memory \& Cognition, 5, 547-552.

Levin, G., \& Baker, B. (1963). Item scrambling in an selfinstructional program. Journal of Educational Psychology, 54, $138-143$.

Marchionni, G. (1988). Hypermedia and learning: Freedom and chaos. Educational Technology, 28, 8-12.

McAleese, R. (1989). Navigation and browsing in hypertext. In R. McAleese (Ed.), Hypertext: Theory into practice (pp.644). 0xford: Intellect Limited. McDonald, S., \& Stevenson, R.J. (1998). Effects of text structure and prior knowledge on the learner on navigation in hypertext. Human Factors, 40, 18-28.

Mcknight, C., Dillon, A., \& Richardson, J. (1990). A comparison of linear and hypertext formats in information retrieval. In. R. McAleese \& C. Green (Eds.), Hypertext: State of the art (pp. 10-19). 0xford: Intellect Limited. 
Mohageg, M. (1992). The influence of hypertext linking structures on the efficiency of information retrieval. Human Factors, 34, 351-367.

Monk, A. (1990). Getting to know locations in a hypertext. In. R. McAleese \& C. Green (Eds.), Hypertext: State of the art (pp. 20-27). Oxford: Intellect Limited.

Norman, D. (1988). The psychology of everyday things. New York: Basic Books.

Rada, R., \& Murphy, C. (1992). Searching versus browsing in hypertext. Hypermedia, 4(1), 1-30.

Rouet, J. (1990). Interactive text processing by inexperienced (hyper)readers. In N. Streitz, A. Rizk \& J. André (Eds.), Hypertext: Concepts, systems and applications (pp. 250260). Cambridge: Cambridge University Press. Shavelson, R., \& Stanton, G. (1975). Concept validation: Methodology and application of three measures of cognitive structure. Journal of Educational Measurement, 12, 67-85. Spiro, R.J., Feltovich, P.J., Jacobson, M.J., \& Coulson, R.L. (1992). Cognitive flexibility, constructivism, and hypertext. Random access instruction for advanced knowledge acquisition in ill-structured domains. In T.M. Duffy \& D.H. Jonassen (Eds.), Constructivism and the technology of instruction: A conversation ( $p p .57-75)$. Hillsdale, NJ: Erlbaum.

Spiro, R.J., \& Jehng, J-.C. (1990). Cognitive flexibility and hypertext: Theory and technology for the nonlinear and multidimensional traversal of complex subject matter. In D. Nix \& R. J. Spiro (Eds.), Cognition, education and 
multimedia: Exploring ideas in high technology ( $p p .163-$ 205). Hillsdale, NJ: Erlbaum.

Tergan, S.-0. (1997). Multiple views, contexts, and symbol systems in learning with hypertext/hypermedia: a critical review of research. Educational Technology, 37(4), 5-18. Tobias, S. (1973). Sequence, familiarity and attribute by treatment interactions in programmed instruction. Journal of Educational Psychology, 64, 133-141.

Vlachouli-Roe, K., Case, H., \& Roe, A. (1962). Scrambled versus ordered sequence in auto-instructional programs. Journal of Educational Psychology, 53, 101-104. de Vries, E., \& de Jong, T. (1997). Using information systems while performing complex tasks an example from architectural design. International Journal of HumanComputer Studies, 46, 31-54. de Vries, E., \& de Jong, T. (1999). Task dependent design and evaluation of information systems; a study into structuring hypertext information for design problem solving, Instructional Science, 27, 285-302.

de Young, L. (1990). Linking considered harmful. In N. Streitz, A. Rizk \& J. André (Eds.), Hypertext: Concepts, systems and applications (pp. 238-249). Cambridge: Cambridge University Press. 
The effects of graphical overviews 31

Figure captions

Figure 1. Part of the interface of the 'visual' condition (Condition1).

Figure 2. Graphical display of the results on the different knowledge tests 
The effects of graphical overviews 32

Table 1

Mean scores (and SD) for connectivity for two phases in the learning process

\begin{tabular}{lcccc}
\hline & \multicolumn{3}{c}{ Initial phase } & Final phase \\
\hline Visual & .95 & $(.08)$ & .90 & $(.18)$ \\
Hints & .92 & $(.16)$ & .86 & $(.09)$ \\
Control & .63 & $(.04)$ & .81 & $(.11)$ \\
\hline
\end{tabular}


The effects of graphical overviews 33

Table 2

Mean scores (and SD) for consistency

\begin{tabular}{lccc}
\hline & \multicolumn{3}{c}{ Conditions } \\
\cline { 2 - 4 } Primitive & $\underline{\text { Visual }}$ & $\underline{\text { Hints }}$ & $\underline{\text { Control }}$ \\
\hline structure & & $.88(.34)$ & $.06(.25)$ \\
\hline Hierarchy & $.93(.26)$ & $.88(.34)$ & $.44(.51)$ \\
Temporal-1 & $.80(.41)$ & $.88(.34)$ & $.25(.45)$ \\
Temporal-2 & $.87(.35)$ & $.69(.48)$ & $.43(.50)$ \\
Causal-1 & $.73(.46)$ & $.81(.40)$ & $.06(.25)$ \\
Causal-2 & $.67(.49)$ & $.56(.51)$ & $.00(.00)$ \\
Causal-3 & $.67(.49)$ & & \\
\hline
\end{tabular}


The effects of graphical overviews 34

Table 3

Mean scores (M) and standard deviations (SD) on the knowledge tests

\begin{tabular}{|c|c|c|c|c|c|c|c|c|c|c|}
\hline \multicolumn{2}{|c|}{ Condition } & \multicolumn{3}{|c|}{ Definitional } & \multicolumn{3}{|c|}{ Propositional } & \multicolumn{3}{|c|}{ Configural } \\
\hline & & Pre & Post & Gain & Pre & Post & Gain & Pre & Post & Gain \\
\hline \multirow[t]{2}{*}{ Visual } & M & 2.67 & 10.00 & 7.28 & 1.53 & 10.33 & 8.08 & -.01 & .49 & .50 \\
\hline & SD & $(2.72)$ & $(3.49)$ & $(3.85)$ & $(1.89)$ & $(3.16)$ & $(3.59)$ & $(.08)$ & $(.32)$ & $(.35)$ \\
\hline \multirow[t]{2}{*}{ Hints } & M & 2.20 & 10.33 & 8.13 & 2.87 & 8.47 & 5.60 & -.02 & .27 & .28 \\
\hline & SD & $(2.14)$ & $(2.29)$ & $(2.95)$ & $(3.42)$ & $(2.00)$ & $(3.85)$ & $(.10)$ & $(.30)$ & $(.41)$ \\
\hline \multirow[t]{2}{*}{ Control } & M & 3.13 & 9.56 & 6.44 & 2.06 & 6.81 & 4.75 & -.02 & .08 & .10 \\
\hline & SD & $(3.05)$ & $(4.12)$ & $(4.53)$ & $(2.27)$ & $(3.19)$ & $(2.65)$ & $(.07)$ & $(.23)$ & $(.22)$ \\
\hline
\end{tabular}




$$
=:=0
$$


The effects of graphical overviews 36
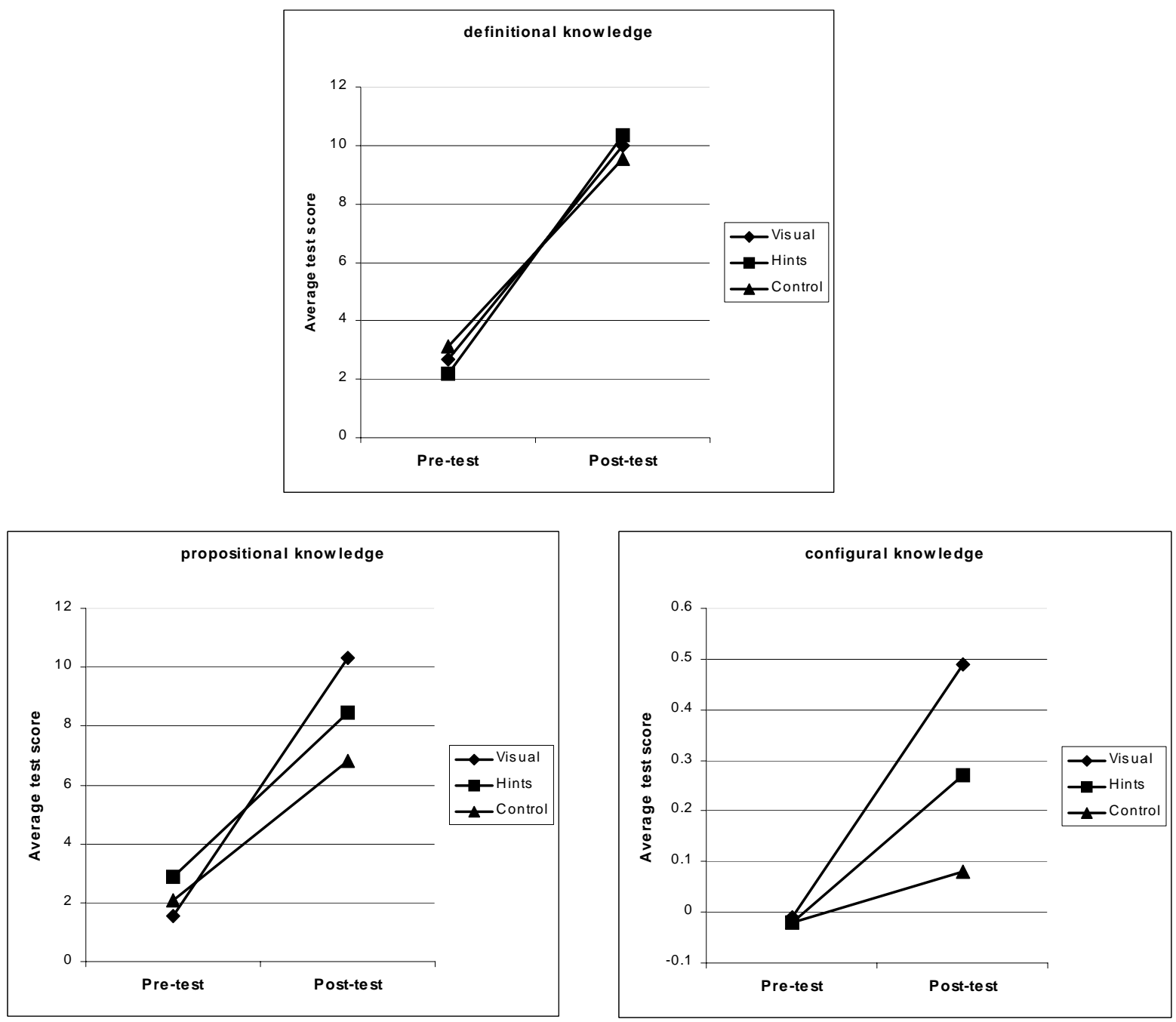

FIGURE 2 